\title{
PANORAMA DA VIOLÊNCIA SEXUAL CONTRA CRIANÇAS E ADOLESCENTES EM MUNICÍPIOS CEARENSES
}

\author{
Overview of sexual violence against children and adolescents \\ in municipalities in Ceará \\ Panorama de la violencia sexual contra niños y adolescentes \\ de municipios de Ceará
}

Artigo Original

\section{RESUMO}

Objetivo: Descrever o panorama da violência sexual contra crianças e adolescentes em municípios do litoral e do sertão do estado do Ceará, Brasil. Métodos: Trata-se de um estudo descritivo realizado em 2014 com análise de dados secundários do "Disque 100" relacionados aos casos de violência sexual contra crianças e adolescentes nos municípios do estado do Ceará, Brasil, referentes ao triênio 2011-2013. Resultados: Foram analisados 210 municípios do sertão cearense, dentre eles o município de Acopiara com 18 (10,8\%) denúncias, sendo destaque para casos de denúncias por abuso sexual contra crianças e adolescentes. Dentre os municípios litorâneos, Caucaia representa o maior número de denúncias, com 112 (35.07\%) casos de abuso sexual. Conclusão: $\mathrm{O}$ estudo é uma alerta às autoridades e à comunidade para atentarem aos casos de violência sexual (exploração e abuso) contra crianças e adolescentes que estão ocorrendo nos diversos municípios cearenses, principalmente na região litorânea.

Descritores: Violência Sexual; Maus-Tratos Infantis; Defesa da Criança e do Adolescente.

\section{ABSTRACT}

Objective: To describe the overview of sexual violence against children and adolescents in coastal municipalities and backlands in Ceará, Brazil. Methods: This is a descriptive study conducted in 2014 with the analysis of secondary data from the "100 Call Service" related to cases of sexual violence against children and adolescents in the municipalities of the state of Ceará, Brazil, for the triennium 2011-2013. Results: A total of 210 municipalities in the backlands of Ceará were analyzed, including the municipality of Acopiara with 18 $(10.8 \%)$ cases, which stood out for the number cases of sexual abuse against children and adolescents. Among the coastal municipalities, Caucaia has the highest number of cases, with 112 (35.07\%) cases of sexual abuse. Conclusion: The study warns government authorities and the community to draw attention to the cases of sexual violence (exploitation and abuse) that are taking place in several municipalities in Ceará, particularly in the coastal region.

Descriptors: Sexual Violence; Child Abuse; Child Advocacy.

\section{RESUMEN}

Objetivo: Describir el panorama de la violencia sexual contra niños y adolescentes en municipios de la costa y de la región agreste del estado de Ceará, Brasil. Métodos: Se trata de un estudio descriptivo realizado en 2014 con análisis de datos secundarios de la "Llamada 100" con relación a los casos de violencia sexual contra niños y adolescentes en los municipios del estado de Ceará, Brasil, referentes al trienio 2011-2013. Resultados: Fueron analizados 210 municipios de la región agreste de Ceará. De estos, el municipio que recibió más casos de denuncias de abuso sexual contra niños y adolescentes fue Acopiara con 18 casos (10,8\%). De los municipios de la costa, Caucaia presentó el mayor número de denuncias con 112 (35,07\%) casos de abuso sexual. Conclusión: El estudio es un alerta para las autoridades y la comunidad para poner atención a los casos de violencia sexual (explotación y abuso) contra niños y adolescentes que ocurren en los diversos municipios de Ceará, principalmente en la región de la costa.

Descriptores: Violencia Sexual; Maltrato a los Niños; Defensa del Niño.

Recebido em: 09/06/2016 Revisado em: 02/07/2016 Aceito em: 30/09/2016
Poliana Hilário Magalhães ${ }^{(1)}$

Deborah Pedrosa Moreira ${ }^{(2)}$

Samira Valentim Gama Lira ${ }^{(1)}$ July Grassiely de Oliveira

Branco $^{(1)}$

Bruna Caroline Rodrigues

Tamboril $^{(1)}$

Luiza Jane Eyre de Souza Vieira $^{(1)}$

1) Universidade de Fortaleza - UNIFOR Fortaleza (CE) - Brasil

2) Centro Universitário UniChristus UniChristus - Fortaleza (CE) - Brasil 


\section{INTRODUÇÃO}

A violência é um fenômeno complexo, por vezes controverso e problemático ${ }^{(1)}$. Ela ocasiona letalidades, lesões, traumas físicos, agravos mentais, emocionais e espirituais. Evidencia a necessidade de uma atuação interdisciplinar, multiprofissional, e intersetorial, visando às necessidades dos cidadãos ${ }^{(2)}$.

O Ministério da Saúde do Brasil, seguindo as recomendações da Organização Mundial de Saúde (OMS) em 1996, declarou que a violência se constitui em importante problema para a saúde pública. Assim, publicou a Política de Redução da Morbimortalidade por Acidentes e Violências ${ }^{(3)}$.

A violência é um tópico constantemente referido na literatura, pois trata de um problema histórico que acompanha a humanidade, apresenta linhagens nas estruturas sociais, políticas e econômicas, bem como nos pensamentos individuais e nas relações sociais. Compreender a violência implica abordá-la e vê-la, também, como uma demanda de saúde pública ${ }^{(4-6)}$, cuja magnitude é crescente, impactando a morbimortalidade das populações, as experiências cotidianas e a reorientação de atitudes e condutas ${ }^{(7,8)}$.

No Brasil, crianças e adolescentes estão entre os grupos mais vulneráveis à violência, sendo a principal causa de morbimortalidade ${ }^{(9)}$. As primeiras manifestações do Estado brasileiro para proteger as crianças e os adolescentes dos eventos de violência ocorreram em 1923 pela Declaração sobre os Direitos da Criança, em Genebra. Porém, somente em 1990, promulga-se o Estatuto da Criança e do Adolescente (ECA), Lei no 8.069, de 13 de julho de 1990(10).

Diante do exposto, torna-se necessário enfatizar que a criminalização de condutas compromete a situações esses grupos vulneráveis que são vítimas de abusos, violências ou explorações de natureza sexual e não se extenuam no Código Penal. Visto que desde sua edição o Estatuto da Criança e do Adolescente tem, em suas contínuas reformulações, se ocupado do tema ${ }^{(11)}$.

As políticas públicas no Brasil, por meio do Sistema Único de Saúde (SUS), recebem o mandato específico do ECA para promover o direito à vida e à saúde de crianças e adolescentes, mediante a atenção integral à saúde, que pressupõe o acesso universal e igualitário aos serviços nos três níveis da atenção. Essa tarefa exige o desenvolvimento de ações de promoção da saúde, prevenção de violências, atenção absoluta às vítimas e o trabalho em rede ${ }^{(12)}$.

A partir de 1993, estabelecem no Brasil algum marco histórico do enfrentamento do abuso sexual contra as crianças e adolescentes. Neste mesmo período, é preparado o relatório da primeira Comissão Parlamentar Mista de Inquérito (CPMI) para averiguar as organizações de exploração sexual de crianças e adolescentes, trazendo à tona numerosos casos de abusos dos direitos infantojuvenis e evidenciar a falta de políticas sociais básicas e de acolhimento às vítimas ${ }^{(13,14)}$.

Com o apoio do Fundo das Nações Unidas para Infância (Unicef), são instituídos em várias cidades do país Centros de Defesa da Criança e do Adolescente (Cedeca), com o intuito de ampliar estrutura de proteção, prevenção e atendimento às crianças, aos adolescentes e aos seus familiares em caso de violência sexual ${ }^{(15)}$.

O Brasil investe nesse campo com a promulgação de políticas públicas voltadas para a proteção de crianças e adolescentes e para o enfrentamento da violência, com destaque para a violência sexual, com vistas a garantir os direitos desse grupo. Sobretudo, nas últimas décadas, não se pode falar de crianças e adolescentes sem que o tema da violência aflore, indicando serem esses dois grupos os mais expostos e vulneráveis a sofrerem violações de seus direitos, afetando direta e indiretamente sua saúde física, mental e emocional ${ }^{(16)}$.

A Secretaria de Direitos Humanos da Presidência da República, em 2003, passou a contar com uma área específica para tratar da prática da violência sexual cometida contra crianças e adolescentes. A partir do Programa Nacional de Enfrentamento da Violência Sexual contra Crianças e Adolescentes (PNEVSCA), iniciativas importantes foram implementadas, como o Disque 100 ou Disque Denúncia Nacional que é um canal de comunicação da sociedade com o poder público e está disponível para todos os estados brasileiros, sendo coordenado e executado pela Secretaria de Direitos Humanos da Presidência da República SDH-PR. Seu principal objetivo é receber denúncias de transgressões aos direitos de crianças e adolescentes e encaminhá-las aos órgãos competentes, além de orientar sobre os serviços e redes de atendimento e proteção para os estados e municípios ${ }^{(12)}$.

O serviço funciona, ainda, como ferramenta epidemiológica da violência no país, contribuindo para a definição de políticas e de áreas prioritárias no atendimento. Apesar de surgir com foco no enfrentamento da violência sexual, o Disque 100 é, hoje, na prática, um Disque Direitos Humanos de Crianças e Adolescentes, acatando denúncias de diferentes tipos de violência envolvendo meninas e meninos $^{(12)}$.

O contexto das grandes cidades e sua relação com a exploração sexual de crianças e adolescentes apresenta um cenário com violação aos direitos humanos, o que gera profundas consequências e desdobramentos prejudiciais a estes sujeitos, sendo discutida em todo o mundo como uma das formas mais extremas de violação dos direitos da criança e do adolescente ${ }^{(17,18)}$.

O Brasil tem empreendido estratégias para enfrentar e prevenir a violência sexual contra crianças e adolescentes. 
Avanços são reconhecidos com o Plano Nacional e a implantação do Programa Sentinela. Entretanto, desajustes minimizam a sua eficácia quanto ao atendimento especializado, destacando-se a deficiência de indicadores que monitorem e avaliem as ações empreendidas nos municípios ${ }^{(19)}$. O setor da educação é parte de uma forma específica de ordenação do mundo que se inicia com a modernidade. Suas raízes estão profundamente interligadas às mudanças que ocorrem a partir deste período. $\mathrm{O}$ modo de organizar o processo, a reconfiguração da família e dos papéis de seus membros, a redefinição e a ampliação da educação escolar foram algumas destas mudanças ${ }^{(20)}$.

Essa iniciativa de pesquisa visa contribuir para melhorar as respostas dos serviços que atuam no enfrentamento da violência sexual mediante as estratégias de ação, como uma ferramenta de gestão pública municipal. A análise proposta servirá para o dimensionamento dos avanços e desafios existentes nos setores da educação com os usuários com vistas à garantia dos direitos desse grupo em situação de violência sexual.

Desta forma, este artigo tem como objetivo descrever o panorama da violência sexual contra crianças e adolescentes em municípios do litoral e do sertão do estado do Ceará, Brasil.

\section{MÉTODOS}

Trata-se de um estudo quantitativo, descritivo com análise de dados secundários relacionados aos casos de violência sexual contra crianças e adolescentes. A solicitação dos dados foi direcionada ao "Disque 100" por meio do cadastro ao sistema por meio do acesso eletrônico "Acesso à Informação", em janeiro de 2014. As informações sobre as denúncias registradas neste sistema foram direcionadas e solicitadas à Secretaria dos Direitos Humanos do Estado do Ceará.

O Disque Denúncia Nacional, ou Disque 100, é um serviço de proteção de crianças e adolescentes com foco em violência sexual, vinculado ao Programa Nacional de Enfrentamento da Violência Sexual contra Crianças e Adolescentes. Trata-se de um canal de comunicação da sociedade civil com o poder público, que possibilita conhecer e avaliar a dimensão da violência e o sistema de proteção, bem como orientar a elaboração de políticas públicas ${ }^{(21)}$.

Após 20 dias da solicitação, os autores receberam os dados em formato de tabelas e gráficos de inúmeras informações referentes à temática violência sexual no Estado do Ceará nos anos de 2011 a 2013. Esses dados foram requeridos ao Governo Federal para ser realizado um diagnóstico da situação epidemiológica da violência sexual, pois no ano de 2014 o país (Brasil) teria um período atípico devido à Copa do Mundo (de Futebol), vivenciando uma realidade distinta dos anos anteriores e adquirindo nos anos posteriores consequências dos atos violentos ocorridos no período de 2014, assim evitando viés nos dados analisados.

Com a posse destes dados, e mediante esta pesquisa ser fruto de um projeto maior que versa sobre o atendimento e a gestão do setor saúde em municípios litorâneos da Região Metropolitana de Fortaleza, Ceará, Brasil, os elementos estudados no presente estudo se relacionam ao panorama dos municípios cearenses (sertão e litoral), em relação às denúncias de violência sexual (exploração e abuso).

Os dados oriundos desta pesquisa foram tabulados e submetidos à análise estatística simples de frequência absoluta e discutida com frequência relativa.

\section{RESULTADOS}

O Estado do Ceará está localizado no Nordeste brasileiro, a taxa de Analfabetismo no Estado diminuiu $29,1 \%$ durante o período $2000 / 2010$, saindo de $26,5 \%$ em 2000 para $18,8 \%$ no ano $2010^{(22)}$.

De acordo com dados da Secretaria de Turismo do Estado (SETUR) no ano de 2012 o Ceará recebeu 2.995.024 turistas via Fortaleza, um incremento de 11,27\% quando comparado ao ano de 2010. Os meses de janeiro, julho e dezembro são historicamente os que mais recebem turistas no Estado. Para o ano de 2012, esses meses juntos corresponderam aproximadamente a $34,6 \%$ do total de turistas. Em um total de $92 \%$ das pessoas que visitaram o Ceará no ano de 2012 foram provenientes do chamado turismo interno, ou seja, 2.761 .413 visitantes vieram dos diversos Estados brasileiros ${ }^{(22)}$.

Analisaram-se os 210 municípios do sertão cearense, dentre eles os municípios que receberam destaque para casos de denúncias por abuso sexual contra crianças e adolescentes foram Acopiara com 18 (10,8\%), Pedra Branca com $17(10,2 \%)$ e Crateús com 15 (9,3\%). Já para os casos de exploração sexual, destaca-se Quixadá e Crateús com 07 $(18,4 \%)$ e $05(13,1 \%)$ respectivamente (Tabela I).

Enfatiza-se que alguns municípios não foram informados nas tabelas, visto que o "Disque 100" não apontou informações sobre eles. Possivelmente, não tiveram denúncias em relação à violência sexual contra crianças e adolescentes.

Conforme a Tabela II, o município de Caucaia representa o município com maior número de denúncias, com 112 casos de abuso sexual, seguido de Jijoca de Jericoacoara com $47(15,1 \%)$ e São Gonçalo do Amarante com $28(8,9 \%)$. Em seguida, com casos de exploração sexual no ranking, estão os municípios de Caucaia, com 28 (26,9\%) casos de denúncias, seguido de Trairi $16(5,1 \%)$ e São Gonçalo do Amarante com 11 (3,5\%).

Rev Bras Promoç Saúde, Fortaleza, 29(3): 414-421, jul./set., 2016 
Tabela I - Caracterização dos municípios do Sertão com denúncias sobre violência contra crianças e adolescentes pelo Disque 100*, no triênio 2011, 2012 e 2013. Fortaleza, Ceará, Brasil, 2014 (n=210).

\begin{tabular}{lcccc}
\hline Município & Abuso Sexual & $\mathbf{\%}$ & Exploração sexual & \% \\
\hline Acopiara & 18 & 10,8 & - & - \\
Pedra Branca & 17 & 10,2 & 01 & 2,6 \\
Crateús & 15 & 9,3 & 05 & 13,1 \\
Tauá & 14 & 8,4 & 04 & 10,5 \\
Quixadá & 12 & 7,2 & 07 & 18,4 \\
Quixeramobim & 11 & 6,6 & 01 & 2,6 \\
Monsenhor Tabosa & 10 & 6,2 & 01 & 2,6 \\
Mombaça & 10 & 6,2 & 01 & 2,6 \\
Parambu & 09 & 5,4 & 01 & 2,6 \\
Senador Pompeu & 08 & 4,8 & 02 & 5,2 \\
Boa Viagem & 05 & 3,1 & 01 & 2,6 \\
Banabuiú & 05 & 3,1 & - & - \\
Solonópole & 05 & 3,1 & - & - \\
Milhã & 04 & 2,4 & 04 & - \\
Novo Oriente & 04 & 2,4 & - & 2,6 \\
Aiuaba & 03 & 1,8 & 01 & - \\
Ararendá & 03 & 1,8 & - & - \\
Catarina & 03 & 1,8 & - & 7,8 \\
Choró & 02 & 1,2 & 03 & 2,6 \\
Nova Russas & 02 & 1,2 & 01 & - \\
Ipaporanga & 02 & 1,2 & - & - \\
Independência & 01 & 0,6 & - & 2,6 \\
Quiterianópolis & 01 & 0,6 & 01 & - \\
Madalena & 01 & 0,6 & 05 & - \\
Deputado Irapuan Pinheiro & 01 & 0,6 & - & -1 \\
\hline Total & 166 & - & 38 & - \\
\hline
\end{tabular}

*Segundo os dados do Governo do Estado do Ceará www.ceara.gov.br

Tabela II - Caracterização dos municípios do Litoral Leste e Oeste com denúncias sobre violência contra crianças e adolescentes pelo Disque 100*, no triênio 2011, 2012 e 2013. Fortaleza, Ceará, Brasil, 2014 (n=417).

\begin{tabular}{lcccc}
\hline Municípios & Abuso Sexual & \% & Exploração sexual & \% \\
\hline Caucaia & 112 & 35,07 & 28 & 26,9 \\
Jijoca de Jericoacoara & 47 & 15,1 & 01 & 0,9 \\
São Gonçalo do Amarante & 28 & 8,9 & 11 & 3,5 \\
Itapipoca & 21 & 6,7 & 07 & 6,7 \\
Aquiraz & 19 & 6,7 & 08 & 2,5 \\
Trairi & 18 & 5,7 & 16 & 5,1 \\
Aracati & 17 & 5,4 & 02 & 1,9 \\
Cascavel & 12 & 3,8 & 09 & 8,6 \\
Beberibe & 11 & 3,5 & 02 & 1,9 \\
Camocim & 9 & 2,8 & 06 & 5,7 \\
Paraipaba & 7 & 2,2 & 03 & 2,8 \\
Paracuru & 6 & 1,9 & 09 & 8,6 \\
Icapuí & 3 & 0,9 & - & - \\
\hline Total & 310 & - & 102 & - \\
\hline
\end{tabular}

*Segundo os dados do Governo do Estado do Ceará - www.ceara.gov.br 
Os dados revelam que no Sertão Cearense os casos de violência sexual (abuso e exploração) ocorrem em menor frequência (204 casos, 33,1\%) do que no Litoral (412 casos, $66,9 \%$ ). Dentre os cinco municípios com principais casos de violência sexual encontra-se Caucaia, São Gonçalo do Amarante e Aquiraz que estão próximos da Capital, favorecendo o acesso de turistas, e, além disso, destacase Jijoca de Jericoacoara que é um município conhecido internacionalmente devido a sua beleza litorânea, ou seja, tudo isso facilita o desenvolvimento do turismo sexual.

\section{DISCUSSÃO}

O aumento das denúncias decorre da recente obrigatoriedade da notificação a casos de abuso sexual infanto-juvenil no país, a partir do Estatuto da Criança e do Adolescente (ECA), Lei 8.069 de $1990^{(10)}$. Em âmbito local, a Secretaria Estadual de Saúde do Ceará determinou a obrigatoriedade da notificação dos casos de maus-tratos contra crianças e adolescentes, implementando ficha própria a ser utilizada em todo o estado ${ }^{(23)}$. Aponta-se para um aumento de denúncias a instituições especializadas no Brasil, como a Associação Brasileira Multiprofissional de Proteção à Infância e à Adolescência (ABRAPIA), os Centros Regionais de Atenção aos Maus-Tratos na Infância (CRAMI) e a Associação Brasileira de Prevenção aos Abusos e Negligências na Infância (ABPANI) ${ }^{(24)}$.

Observa-se, após análise dos dados da presente pesquisa, que a quantidade de denúncias realizadas dos municípios do litoral (402) é quase o dobro às realizadas nos sertanejos (204), apesar de se apresentar um quantitativo maior de municípios no sertão que no litoral.

No Brasil, uma das representações da violência sexual produz-se por meio do turismo e da pornografia, com maior frequência nas capitais do Nordeste e outros centros ${ }^{(25)}$. A produção do turismo convida os usuários desse setor a desfrutarem de múltiplas opções, o que beneficia a produção e reprodução dos serviços. Além disso, os roteiros das viagens programados por empresas de âmbito global dificultam a vigilância neste setor, uma vez que os pacotes turísticos possuem uma organização compacta de serviços e, geralmente, são finalizados de forma real ou virtual( ${ }^{(25,26)}$.

Destaca-se, entretanto, que os municípios do litoral do estado do Ceará contam com municípios mais populosos, como os da Região Metropolitana como Caucaia (município de grande porte). Segundo dados do $\operatorname{IBGE}^{(27)}$, a população de crianças e adolescentes residentes em Caucaia, no ano de 2010, chegava a 122.956 , representando o percentual de $37,7 \%$ do total de habitantes (325.411). Além disso, conta com dois Conselhos Tutelares.
A sociedade brasileira e a mundial há muitos anos vêm enfrentando problemas relacionados ao abuso e exploração sexual contra menores. Infelizmente, o Brasil possui vários fatores para que esse mal prevaleça na sociedade, tais como: falta de uma educação de qualidade que venha a informar e educar essas crianças e adolescentes, juntamente com seus familiares; a pobreza; a miséria; a violência e a má distribuição de renda; todos esses fatores elevam o grau de possibilidade para que esse ato venha a ser realizado. Apesar dos altos índices relacionados à temática em questão, o abuso e a exploração sexuais vem ganhando maior respaldo pelas políticas públicas e pela sociedade em geral $^{(28)}$.

A violência sexual (exploração e abuso) no contexto do turismo, enquanto violação aos direitos, não pode ser entendida como a vinda de pessoas estrangeiras ao território brasileiro/cearense com o objetivo de se relacionarem sexualmente com brasileiros ou brasileiras, mas, sim, como a vinda dessas pessoas visando à realização de alguma conduta que constitua turismo sexual, que muitas vezes são facilitadas por agências de viagens, restaurantes, bares e hotéis. Essa realidade está presente nas diversas regiões brasileiras, estando em destaque a região Nordeste devido ao seu caráter turístico ${ }^{(24,29)}$.

No caso em que haja imagens de crianças ou adolescentes, configura-se como abuso ou exploração, já que não se trata de imagens de pornografia, e sim de um crime/violência praticado. O ECA também prevê crimes de exploração sexual em seus artigos 240 a $243^{(10)}$.

No âmbito das políticas públicas governamentais que tratam especificamente do abuso e exploração sexual de menores, temos: o Programa Sentinela e o PAIR (Programa de Ações Integradas e Referenciais de Enfrentamento à Violência Sexual Infanto-Juvenil), atualmente sendo implantados em Imperatriz, esses programas são do Governo Federal em parceria com estados e municípios ${ }^{(30)}$.

O Programa Sentinela foi elaborado para desempenhar o papel de atender as determinações da Constituição Federal, do Estatuto da Criança e do Adolescente, da Lei Orgânica de Assistência Social e do Programa Nacional de Enfrentamento à Violência Sexual Infanto-juvenil, atuando nos eixos de atendimento, mobilização e articulação, prevenção, defesa e responsabilização ${ }^{(10,30)}$.

O Programa Sentinela constitui-se numa ação de responsabilidade do Ministério do Desenvolvimento Social e Combate à Fome - MDS -, inserido no programa ao Abuso e Exploração Sexual de Crianças e Adolescentes, coordenado pela Secretaria Especial dos Direitos Humanos da Presidência da República e está atualmente presente em todos os estados da federação e no Distrito Federal. Em 2006, com a implantação do Sistema Único de Saúde 
- SUS -, o Sentinela se insere como serviço do Centro de Referência de Assistência Social - CAS -, obedecendo às normas operacionais básicas da política pública de Assistência Social, alcançando uma abrangência de 1104 municípios brasileiros ${ }^{(31)}$.

O "abuso sexual" muitas vezes não deixa rastro, ou seja, não deixa marcas de violência física e também pelo fato de esse ato ser praticado dentro do próprio lar e por pessoas que têm relação de parentesco com a vítima. Assim, torna-se mais difícil detectar o problema, pois quando a mãe ou alguém da família vem tomar conhecimento da situação, isso pode estar acontecendo há muito tempo, então o "estrago psicológico" dessa criança na maioria das vezes é irremediável ${ }^{(32)}$.

A violência sexual no Brasil é algo alarmante, em vista disso, este fenômeno foi incluído na agenda da sociedade civil em prol da luta nacional e internacional pelos direitos humanos da criança e do adolescente, contidas na Constituição Federal Brasileira de 1988, no Estatuto da Criança e do Adolescente, Lei 8.069/90 ${ }^{(10)}$. O PAIR ${ }^{(33)}$ é um programa do Governo Federal em parceria com a Agência Norte-Americana para o Desenvolvimento Internacional USAID.

Vale ressaltar que estas instituições acima mencionadas, Sentinela e PAIR, trabalham em parceria com o Conselho Tutelar, órgão municipal que possui como função atender a casos de violência e à violação dos direitos da criança e adolescente; a casa de passagem, órgão do governo municipal, onde ficam temporariamente os menores vítimas da violação de seus direitos; a delegacia da mulher, devido à falta de uma delegacia de proteção à infância e à juventude na cidade de Imperatriz e o Ministério Público, órgão que também defende os direitos da criança e do adolescente ${ }^{(33)}$.

Nesse contexto, destaca-se a Rede Aquarela que é um programa que executa e coordena ações de enfrentamento da violência sexual contra crianças e adolescentes da cidade de Fortaleza. Vinculado à Coordenadoria da Criança e do Adolescente e a Secretaria de Cidadania dos Direitos Humanos/FUNCI, a rede teve como objetivo inicial o desenvolvimento de ações de maior amplitude a partir de uma efetiva articulação para minimizar os casos de violência sexual ${ }^{(30)}$.

Desta forma, o enfrentamento da violência sexual exige uma série de ações articuladas em diversos setores. A Rede Aquarela pauta essas ações nas diversas instâncias na cidade de Fortaleza, onde poder público e sociedade civil estão articulados realizando ações intersetoriais de mobilização, promoção de direitos e do controle social. A rede estabelece uma agenda de mobilização pautada, principalmente, em campanhas e formações. Para a consolidação dessa política, a articulação acontece com o Governo do Estado do Ceará;
Ministério Público; Delegacia de Combate à Exploração de Crianças e Adolescentes (DECECA); Saúde; Escolas; Conselhos Tutelares e de Direitos; Organizações NãoGovernamentais; associações comunitárias e igrejas entre outros $^{(6)}$.

O Sistema de Vigilância de Violências e Acidentes em Serviços Sentinelas -VIVA mostra que crianças e adolescentes independente de gênero são igualmente vulneráveis a sofrer violência, embora sejam afetados por distintos tipos e expressões de situações violentas ${ }^{(33)}$.

Os direitos das crianças repousam no ordenamento jurídico brasileiro e que, às vezes, são advertidos pela violência enraizada na contextura social, que afeta notadamente os mais vulneráveis. A violência não somente resulta em altos custos econômicos e sociais para a sociedade, mas também tem profundas implicações emocionais nas linhagens, devido ao impacto na saúde e na qualidade de $\operatorname{vida}^{(12)}$.

Diante dos dados analisados e disponibilizados pelo Disque 100, o presente estudo apenas conseguiu analisar a frequência da violência sexual (exploração e abuso) nos anos de 2011 a 2013, não podendo correlacioná-la com outros fatores, devido ao não acesso aos dados, limitando assim as inferências acerca desses atos violentos. Mas, é possível o questionamento sobre como esses municípios podem estar realizando atividades para o enfrentamento desses casos, pois quando uma criança/adolescente é violentada, muitas vezes envolve seus familiares, necessitando de um suporte dos diversos serviços que garantam os direitos desse grupo.

\section{CONCLUSÃO}

O estudo é uma alerta às autoridades do governo e à comunidade para atentarem aos casos de violência sexual (exploração e abuso) que estão ocorrendo nos diversos municípios Cearenses, principalmente na região litorânea, onde a maioria da população acaba tendo mais acesso e em contrapartida, sendo mais divulgada como 'propaganda' no intuito de atrair turistas, melhorando o turismo e a gestão do município. Então, é necessário que diversas estratégias sejam realizadas para o enfrentamento dessa casuística, para minimizar esses casos.

\section{REFERÊNCIAS}

1. Deslandes SF. Sumário de pesquisa: avaliação das estratégias governamentais municipais no enfrentamento da violência sexual e exploração sexual de crianças e adolescentes em quatro capitais brasileiras. Rio de Janeiro: FIOCRUZ/ENSP/IFF; 2013. 
2. Deslandes SF, Cavalcanti LF, Vieira LJES, Silva RM. Capacitação profissional para o enfrentamento às violências sexuais contra crianças e adolescentes em Fortaleza, Ceará, Brasil. Cad Saúde Pública. 2015;31(2):431-5.

3. Ministério da Saúde (BR), Secretaria de Vigilância em Saúde, Departamento de Análise de situação de saúde: política nacional de redução da morbimortalidade por acidentes e violências. Brasília: Ministério da Saúde; 2005 .

4. Organização das Nações Unidas - ONU. Assembléia Geral das Nações Unidas. Convenção das Nações Unidas sobre os Direitos da Criança. 1989 [acesso em 2015 Set 18]. Disponível em: http://www.onubrasil. org.br/doc_crianca.php

5. Krug EG, Dahlberg LL, Mercy JA, Zwi AB, Lozano R. World report on violence and health. Geneva: WHO; 2002.

6. Ministério da Saúde (BR), Secretaria de Atenção à Saúde, Área de Saúde do Adolescente e do Jovem: Marco legal: saúde, um direito de adolescentes. Brasília: Ministério da Saúde; 2005.

7. Santos VA, Costa LF. A violência sexual contra crianças e adolescentes: conhecer a realidade possibilita a ação protetiva. Estud Psicol. 2011;28(4):529-37.

8. Batista KBC, Gonçalves OSJ. Formação dos profissionais de saúde para o SUS: significado e cuidado. Saúde Soc. 2011;20(4):884-99.

9. Ministério da Saúde (BR). Mortalidade por causas externas [Internet]. Brasília: Ministério da Saúde; 2014 [acesso em 2016 Abr 27]. Disponível em http://tabnet. datasus.gov.br/cgi/tabcgi.exe?sim/ cnv/obt10uf.def.

10. Brasil. Lei Federal no 8069 de 1990. Dispõe sobre o Estatuto da Criança e do Adolescente e dá outras providências. Diário Oficial da República Federativa do Brasil, 1990 Jul 13 [acesso em 2016 Mar 5]. Disponível em: http://www.planalto.gov.br/ccivil_03/ Leis/L8069.htm.

11. Veronese JRP. Violência e exploração sexual infanto-juvenil: uma análise conceitual. Psicol Clín. 2012;24(1):117-33.

12. Ministério da Saúde (BR), Secretaria de Atenção à Saúde, Departamento de Ações Programáticas Estratégicas. Linha de cuidado para a atenção integral à saúde de crianças, adolescentes e suas famílias em situação de violências: orientação para gestores e profissionais de saúde. Brasília: Ministério da Saúde; 2010 .
13. Trabbold VLM, Caleiro CRL, Cunha CF, Guerra AMC. Concepções sobre adolescentes em situação de violência sexual. Psicol Soc. 2016;28(1):74-83.

14. Leal MLP. As ONGS no enfrentamento da exploração, abuso sexual e maus tratos de crianças e adolescente Pós 1993. Ser Social. 1998;2:131-156.

15. Comitê Nacional de Enfrentamento da Violência Sexual Contra Crianças e Adolescentes. Marcos históricos do enfrentamento da violência sexual contra crianças e adolescentes. Brasília. [acesso em 2014 Nov 6]. Disponível em: http://www.sdh.gov.br/ assuntos/bibliotecavirtual/criancas-e-adolescentes/ publicacoes-2013/pdfs/plano-nacional-deenfrentamento-da-violencia-sexual-contra-crianca-eadolescentes

16. Souza ER, Jorge MHPM. Impacto da violência na infância e adolescência brasileiras: magnitude da morbimortalidade. In: Lima CA (Org.). Violência faz mal à saúde. Brasília: Ministério da Saúde; 2006. p. 23-8.

17. Nunes AJ, Sales MCV. Violência contra crianças no cenário brasileiro. Ciênc Saúde Coletiva. 2016;21(3):871-80.

18. Childhood. Relatório de atividades [Internet]. São Paulo; 2013 [acesso em 2015 Nov 6]. Disponível em: http:/www.childhood.org.br/ wpcontent/uploads/2014/10/2310/PDFinterativo_ CHILDHOOD_PORT.pdf

19. Paixao ACW, Deslandes SF. Abuso sexual infanto juvenil: ações municipais da Saúde para a garantia do atendimento. Ciênc Saúde Coletiva. 2011;16(10): 4189-98.

20. Alves R. Violência na escola e da escola: desafios contemporâneos à Psicologia da Educação. Psicol Esc Educ. 2009;13(2):343-6.

21. Ministério da Justiça e Cidadania (BR), Secretaria Especial de Direitos Humanos. Disque 100: Disque direitos humanos [acesso em 2014 Nov 24]. Disponível em: http:/www.sdh.gov.br/disque-direitos-humanos/ disque-direitos-humanos

22. IPECE. Instituto de Pesquisa e Estratégia Econômica do Ceará, 2013. [acesso em 2016 Ago 4]. Disponível: http://www.ipece.ce.gov.br/

23. Ministério da Saúde (BR), Secretaria de Vigilância em Saúde. Sistema nacional de vigilância em saúde: relatório de situação: Ceará. Brasília: Ministério da Saúde; 2011. 
24. Moreira DP. Concepções de gestores da saúde sobre violência sexual, contra crianças e adolescentes [tese]. Fortaleza: Universidade de Fortaleza; 2015.

25. Silva TA. A exploração sexual de crianças e adolescentes no turismo: uma análise da atuação da Rede de Enfrentamento em Porto Seguro [dissertação]. Ilhéus: Universidade Estadual de Santa Cruz; 2009.

26. Araújo EF, Dantas EWC. As políticas públicas e o turismo litorâneo na Região Metropolitana de Fortaleza - CE. Sociedade Território. 2011;23(2):57-73.

27. Instituto Brasileiro de Geografia e Estatística - IBGE. Síntese de Indicadores Sociais. 2010. [acesso em 2014 Fev 15]. Disponível em: http://www.ibge. gov.br/home/estatistica/populacao/condicaodevida/ indicadoresminimos/sinteseindicsociais2010/

28. Moreira DP, Vieira LJES, Pordeus AMJ, Lira SVG, Luna GLM, Silva JG. Exposição à violência entre adolescentes de uma comunidade de baixa renda no Nordeste do Brasil. Ciênc Saúde Coletiva. 2013;18(5):1273-82.

29. Figueiredo K, Bocchi SB. Violência sexual: um fenômeno complexo [Internet]. Brasília: Unicef; 2010. [acesso em 2015 Mar 21]. Disponível em: https://www. unicef.org/brazil/pt/Cap_03.pdf
30. Ministério da Saúde (BR). Política Nacional de Promoção da Saúde: Portaria MS/GM n. 687, de 30 de março de 2006. Brasília: Ministério da Saúde; 2006.

31. Aragão AS. Rede de proteção social e promoção de direitos: contribuições do conselho tutelar para a integralidade e a intersetorialidade [tese]. Ribeirão Preto (SP): Universidade de São Paulo; 2011.

32. Assis SG, Avanci JQ, Pesce RP, Pires TO, Gomes DL. Notificações de violência doméstica, sexual e outras violências contra crianças no Brasil. Ciênc Saúde Coletiva. 2012;17(2):2305-17.

33. Ministério da Justiça e Cidadania (BR), Secretaria Especial de Direitos Humanos. Programa de Ações Integradas e Referenciais de Enfrentamento à Violência Sexual Infanto juvenil no Território Brasileiro (PAIR). [acesso em 2014 Out 15]. Disponível em: http://pair. ledes.net/

\section{Endereço para correspondência:}

Poliana Hilário Magalhães

Universidade de Fortaleza - UNIFOR

Programa de Pós-Graduação em Saúde Coletiva - PPGSC

Avenida Washington Soares, 1321

Bairro: Edson Queiroz

CEP: 6060811-905 - Fortaleza - CE - Brasil

E-mail: polyanahm@hotmail.com 